\title{
TERMORREGULAÇÃO ESCROTAL EM TOUROS NELORE (Bos taurus indicus)
}

Camila Dutra de Souza ${ }^{1}$, Marcelo George Mungai Chacur ${ }^{1}$, Felipe Rydygier de Ruediger ${ }^{1}$, Júnia de Souza Cartocci ${ }^{1}$, Isamara Batata Andrade ${ }^{1}$, Guilherme Pepino Bastos ${ }^{1}$, Eunice Oba ${ }^{2}$, Alcides Amorim Ramos ${ }^{2}$

${ }^{1}$ Universidade do Oeste Paulista - UNOESTE, Faculdade de Medicina Veterinária, Laboratório de Reprodução Animal, Presidente Prudente - SP. ${ }^{2}$ Universidade Estadual Paulista - UNESP, Faculdade de Medicina Veterinária, Laboratório de Reprodução Animal, Botucatu - SP, Brasil. E-mail: camiladutrasouza@hotmail.com

\section{RESUMO}

O objetivo desse estudo foi determinar a temperatura superficial do escroto com o uso da câmera de termografia por Infravermelho em touros Nelore criados extensivamente, antes e após a colheita do sêmen. Foram utilizados 80 touros Nelore com 24 a 36 meses, nos quais foi coletado o sêmen por eletroejaculação e imagens termográficas por infravermelho do escroto antes e após a colheita. Os dados foram avaliados por análise de variância e comparação de médias pelo teste TUKEY a $5 \%$. Os resultados mostraram que $(P<0,05)$ entre os pontos anatômicos analisados na mesma imagem termográfica (cordões espermáticos em relação aos pontos dorsal, médio e ventral dos testículos; e cauda dos epidídimos) tanto antes quanto após a colheita do sêmen. No entanto, $(P>0,05)$ quando comparadas as médias de cada ponto anatômico entre as imagens antes e depois da colheita. Em conclusão, a termografia por Infravermelho pode ser realizada antes ou após a colheita do sêmen e em condições de campo, sendo um auxílio à seleção de reprodutores quando adicionada ao exame andrológico.

Palavras-chave: zebu; imagem termográfica; termografia por infravermelho; sêmen; temperatura escrotal

\section{SCROTAL THERMOREGULATION IN NELORE BULLS (Bos taurus indicus)}

\begin{abstract}
The aim of this study was to determine the scrotal surface temperature with the use of infrared thermography camera in Nellore bulls under field conditions, before and after collection of semen. 80 Nelore bulls with 24-36 months were used, in which the semen was collected by electroejaculation and infrared thermographic images of the scrotum before and after collection of semen. Data were evaluated by analysis of variance and comparison of means by Tukey test at $5 \%$. The results showed that $(P<0.05)$ between the anatomical points analyzed in the same thermographic image (spermatic cords in relation to dorsal, middle and ventral points of the testicles, and at the cauda of the epididimides) both before and after collection of semen. However, ( $P>0.05$ ) when comparing the mean of each anatomical point between the images before and after harvest. In conclusion, Infrared thermography can be performed before or after semen collection and field conditions, being an aid to the selection of breeding soundness examination when added to.
\end{abstract}

Keywords: zebu; thermographic images; infrared thermography; semen; scrotal temperature. 


\section{INTRODUÇÃO}

No Brasil, estudos sobre a interferência ambiental nas características físicas e morfológicas espermáticas de touros têm sido conduzidos em condições de campo por Chacur et al. (2012) e em centrais de inseminação artificial (IA), com animais em regime permanente de colheita de sêmen (BRITO et al., 2002).

Diversos fatores afetam a produção animal, entre eles a temperatura, umidade, nutrição, doenças, parasitas e alterações ambientais de diferentes origens, numa revisão de literatura sobre a reprodução de bovinos nos trópicos, Galina e Arthur (1991) abordaram a importância da variação sazonal sobre as características físicas e morfológicas espermáticas, relatando que a baixa qualidade do sêmen em alguns animais pode ocorrer devido ao desconforto destes perante temperaturas elevadas.

A temperatura testicular em touros deve ser 4 a $5^{\circ} \mathrm{C}$ abaixo da temperatura corporal para que ocorra espermatogênese normal (COULTER, 1988). Segundo Kastelic et al. (2001), uma moderada elevação da temperatura testicular em touros submetidos à insulação escrotal reduz drasticamente a produção espermática, a motilidade progressiva, a quantidade de espermatozóides vivos por ejaculado e aumenta a porcentagem de espermatozóides morfologicamente anormais. Mudanças climáticas que afetam a gametogênese podem levar a baixa eficiência reprodutiva de touros (VALE FILHO, 2001; CHACUR et al., 2012).

A utilização da termografia por infravermelho é uma técnica que pode auxiliar na identificação do estresse térmico dos animais. A análise de imagens termográficas por infravermelho, onde é possível visualizar gradientes de temperatura, tem sido utilizada para identificar eventos fisiológicos em animais e humanos (BOUZIDA et al., 2009).

A termografia por Infravermelho é um método não invasivo de avaliação da temperatura superficial do escroto por emissão infravermelha com uma acurácia de 0.10 ㄷ․ Pesquisas têm mostrado que a temperatura superficial do escroto está altamente correlacionada com a temperatura interna dos testículos (COULTER et al., 1988). Não existe efeito significativo da variação diurna sobre a temperatura superficial do escroto.

Considerando a predominância da criação extensiva e a monta natural é importante investigar a influência dos fatores climáticos sobre as características seminais de touros mantidos no campo. O presente trabalho se justifica pelo pequeno enfoque em relação ao uso da termografia em animais zebuínos. 
O objetivo desse estudo foi determinar a temperatura superficial do escroto com a Câmera de Termografia por Infravermelho em touros Nelore criados extensivamente, antes e após a colheita do sêmen.

\section{METODOLOGIA}

A presente pesquisa foi encaminhada, avaliada e aprovada pelo Comitê de Ética em Uso de Animais com o protocolo № 1920.

O experimento foi realizado em uma propriedade rural pertencente ao município de Campo Grande - MS, latitude Sul $20^{\circ} 26^{\prime} 34^{\prime \prime}$ S, e Longitude W.GE $54^{\circ} 38^{\prime} 45^{\prime \prime}$ W, com precipitação média de $1225 \mathrm{~mm} / a n o$, temperatura média anual de 29으, clima tropical com estação seca. Dessa propriedade foram utilizados 80 touros da raça Nelore, com idades entre 24 e 36 meses, criados extensivamente, em pasto de Brachiaria decumbens, com acesso ao sal mineral e água ad libitum.

O sêmen dos touros foi coletado por eletroejaculação (Autoejac ${ }^{\circledR}$, Neovet), com aparelho automático, proporcionando maior conforto aos animais durante a colheita. A uma temperatura ambiente média de $31^{\circ} \mathrm{C}$ sob condições a campo. Para cada macho foi coletado uma amostra de sêmen, totalizando 80 amostras.

As amostras de sêmen foram mantidas em banho-maria, entre 32 e $35^{\circ} \mathrm{C}$, para as análises imediatas da motilidade espermática progressiva, vigor espermático e turbilhão. Com posterior diluição do sêmen em formol salino tamponado (1:100), para obtenção da concentração espermática em câmara de Neubauer e confeccionada um esfregaço do sêmen para posterior análise da morfologia espermática frente à avaliação de 200 células com microscopia óptica de contraste de fase. Os touros foram classificados segundo as avaliações clínicas e espermáticas para efeito de seleção para monta natural, segundo as normas do Colégio Brasileiro de Reprodução Animal (CBRA, 1998).

A mensuração do comprimento, largura e altura dos testículos foi realizada com o auxílio de um paquímetro. $\mathrm{O}$ perímetro escrotal $(\mathrm{PE})$ foi mensurado com fita métrica graduada em centímetros, aferido na região de maior diâmetro do escroto. Para o cálculo do volume testicular (VT), será utilizada a expressão: VT =0,0396 $\times$ (média do comprimento dos testículos) $\times$ (perímetro escrotal) ${ }^{2}$, conforme Lunstra et al. (1988).

A câmera de termografia por Infravermelho (FLIR E-40 $\left.{ }^{\circledast}\right)$ foi posicionada a $1 \mathrm{~m}$ do escroto para a confecção da imagem de termografia por Infravermelho. Foram capturadas duas imagens para cada touro, onde uma foi confeccionada antes da colheita do sêmen e a outra, após a 
colheita. A câmera de termografia por Infravermelho possui um software de computador, que nos permitiu a análise da imagem, marcando pontos específicos nas mesmas. Nas imagens do escroto de cada animal foram marcados os pontos: cordões espermáticos (T1), no ponto dorsal do testículo (T2), no ponto médio do testículo (T3), no ponto ventral do Testículo (T4) e na cauda dos epidídimos (T5) em cada um dos testículos.

O gradiente de temperatura escrotal (GTE) foi obtido pela diferença da temperatura da superfície na região do colo do escroto em relação às temperaturas nos terços dorsal (TTD), médio (TTM) e ventral (TTV) do escroto, por meio da análise das imagens (termogramas), segundo Lunstra e Coulter (1997).

Os dados foram avaliados por análise de variância e comparação de médias pelo teste de Tukey com nível de significância de 5\%.

\section{RESULTADOS}

As médias das variáveis das amostras de sêmen analisadas foram: volume do ejaculado (5.48 $\pm 3.08 \mathrm{~mL})$, motilidade espermática progressiva $(73.44 \pm 15.28 \%)$, vigor espermático (3.44 \pm 0.86$)$, turbilhão $(3.12 \pm 0.78)$ e defeitos espermáticos totais $(21.02 \pm 16.43 \%)$. E com um volume testicular médio de $\left(708.19 \pm 164.16 \mathrm{~cm}^{3}\right)$. Esses resultados das variáveis analisadas demonstram a boa qualidade do sêmen.

As médias de T1 a T5 antes da eletroejaculação foram diferentes $(P<0,05)$, onde T1

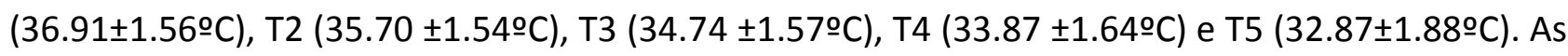
médias de T1 a T5 depois da eletroejaculação foram diferentes $(P<0,05)$, onde $T 1(36.72 \pm 1.70$ 으),

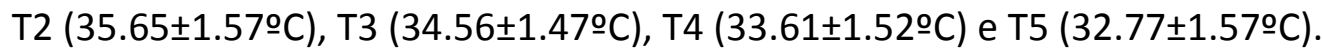

Comparando a condição antes com a depois da eletroejaculação, não existe diferença $(P>0,05)$ entre as temperaturas dos mesmos pontos anatômicos dos testículos (T1 antes - T1 depois; T2 antes - T2 depois; T3 antes - T3 depois; T4 antes - T4 depois; T5 antes - T5 depois).

\section{DISCUSSÃO}

Com esse experimento foi observado que no gradiente de temperatura, a subtração entre os pontos T1 antes e T5 antes, observe-se um resultado de 4.04으. Assim como em T1 depois - T5 depois, que observe-se o resultado de 3.95으. Esse resultados concordam com Coulter (1988) que em seu experimento obteve os padrões de temperatura de touros com a termorregulação escrotal normal tinham simetria da esquerda para direita e as temperaturas se mostraram cerca de 40 C mais altas no topo do que na extremidade distal do escroto. 
Lunstra e Coulter (1997) relataram que os animais com gradiente de temperatura de $2,6 \pm 0,1$ 으 entre a porção alta do escroto e a porção medial do mesmo, produzem sêmen com

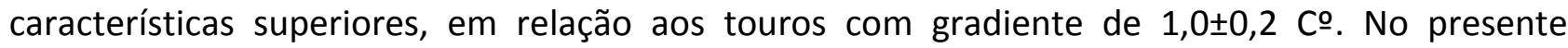
experimento, obteve-se um gradiente de temperatura antes da colheita de $2.17 \circ \mathrm{C}$ e após a colheita de 2.16ㄷ $\mathrm{C}$ para os pontos T1 antes e T3 antes, e T1 depois e T3 depois, respectivamente. E esses gradientes relacionados à qualidade seminal estudada no presente estudo, revelam que essa diferença encontrada também pode estar associada à um sêmen com características superiores.

A termografia, no Brasil, foi empregada em zebus da raça Nelore por Silva et al. (2011) durante os meses de outubro e novembro (primavera), aferindo a temperatura retal (TR) e as temperaturas no colo do escroto (T1) e terços dorsal (T2), médio (T3) e ventral (T4) do escroto; e da região da cauda do epidídimo (T5) com termômetro de infravermelho, obtendo temperatura média de $33,9 \pm 0,4$ ํ $\mathrm{C}$ nos testículos (T2, T3 e T4) e de $30,4 \pm 0,3{ }^{\circ} \mathrm{C}$ nos epidídimos (T5).

\section{CONCLUSÃO}

A termografia por Infravermelho pode ser realizada antes ou após a colheita do sêmen e pode ser utilizada em condições de campo. A termografia por Infravermelho revelou acurácia nas mensurações com decréscimo das temperaturas do cordão espermático (T1) em relação ao testículo (T2, T3 e T4), e destes em relação a cauda do epidídimo (T5).

\section{REFERÊNCIAS}

BOUZIDA, N; BENDADA, A; MALDAGUE, X. P. Visualization of body thermoregulation by infrared imaging. Journal Thermal Biology; v. 34, n.3, p. 120-126, 2009.

http://dx.doi.org/10.1016/j.jtherbio.2008.11.008

BRITO, L.C.F.; SILVA, A. E. D. F.; RODRIGUES, L. H.; VIEIRA, F.V.; DERAGON, L.A.G., KASTELIC, J.P. Effects of environmental factors, age and genotype on sperm production and semen quality in Bos indicus and Bos taurus Al bulls in Brazil. Animal Reproduction Science, v. 70, n.1, p.181-190, 2002. http://dx.doi.org/10.1016/S0378-4320(02)00009-X

CHACUR, M.G.M.; MIZUSAKI, K.T.; SANTOS, F.H.; CESARE, A.G.; GABRIEL FILHO, L.R.A.; OBA, E.; RAMOS, A.A. Influence of year season on semen characteristics and hormonal levels in Nellore and Simmental bulls. Arquivo Brasileiro de Medicina Veterinária e Zootecnia, v.64, n.2, 2012.

COLÉGIO BRASILEIRO DE REPRODUÇÃO ANIMAL (CBRA). Manual para exame andrológico e avaliação de sêmen animal. 2.ed. Belo Horizonte, 1998. 49p.

COULTER, G.H. Thermography of bull testes. Proc. $12^{\text {th }}$. Tech. Conf. Artif. Insemin. \& Reprod. (Natl. Assoc. Anim. Breeders) p.58-62, 1988. 
COULTER, G.H.; SENGER, P.L.; BAILEY, D.R.C. Relationship of scrotal surface temperature mensured by infrared thermography to subcutaneous and deep testicular temperature in the ram. J. Reprod. Fertil., v.84, p.417-423, 1988. http://dx.doi.org/10.1530/jrf.0.0840417

GALINA, C.S.; ARTHUR, G.H. Review of cattle reproduction in tropics. Part 6. The Male. Animal Breeding Abstracts, v.59. p.403-412, 1991.

KASTELIC, J.; COOK, R.B.; PIERSON, R.A.; COULTER, G.H. Relationships among scrotal and testicular characteristics, sperm production and seminal quality in 129 beef bulls. Canadian Journal of Veterinary Research, Ottawa, n.65, p.111-115, 2001.

LUNSTRA, D.D.; COULTER, G.H. Relationship between infrared temperature patterns and naturalmating fertility in beef bulls. Journal of Animal Science, v.75, p.767-774, 1997. http://dx.doi.org/10.1016/0093-691X(88)90270-1

LUNSTRA, D.D.; GREGORY, K.E.; CUNDIFF, L.V. Heritability estimates and adjustment factors for effects of bull age and age of dam on yearling testicular size in breeds of beef bulls. Theriogenology, v.30, p.127-136, 1988.

SILVA, A.A.; CHACUR, M.G.M.; MARTINS, E.A.; AGOSTINHO, B.F.; MARTINS, M.C.; OBA, E. Scrotal infrared thermometry pre and post semen collect in Nelore bulls. Revista Brasileira de Reprodução Animal, v.35, n.2, 2011.

VALE-FILHO, V.R. Subfertilidade em touros: parâmetros para avaliação andrológica e conceituação geral. Caderno Técnico de Veterinária e Zootécnica. Belo Horizonte, n.35, p.81-87, 2001. 